\title{
UPPER SEMICONTINUOUS DECOMPOSITIONS OF DEVELOPABLE SPACES ${ }^{1}$
}

\author{
JOHN M. WORRELL, JR.
}

Presented here are theorems concerning upper semicontinuous decompositions of developable spaces, topological in the sense that the common parts of intersecting domains (open sets) are open. Theorem 1 shows that, if the elements of such a decomposition do not have nonbicompact [1] intersections with the closures of their complements, the decomposition space is developable. Theorem 2 shows that if additionally the space covered by the decomposition is complete in a certain Cauchy sense defined below the decomposition space is complete in this sense. Theorem 3 is a variation of Theorem 2 dealing with a nonequivalent [10] Ascoli type completeness property. Under Consequences some implications of Theorems 1 and 2 are given. One of these gives affirmative resolutions of the following questions raised by R. L. Moore: (1) Do upper semicontinuous decompositions into compact point sets of spaces satisfying Axiom 0 and Axiom $1_{3}$ (the first three conditions of Axiom 1) of "Foundations of point set theory" [6] yield spaces satisfying these axioms? (2) Do such decompositions of spaces satisfying Axiom 1 yield spaces satisfying this axiom? Two other consequences are theorems of Morita-Hanai-Stone [7], [12] and I. A. Vaĭnšteĭn [13].

The sequence $G_{1}, G_{2}, G_{3}, \cdots$ is said to be a development of the space $\Sigma$ provided that (1) for each $n, G_{n}$ is a collection of domains covering $\Sigma$ and (2) if $P$ is a point and $D$ is a domain containing $P$, then for some $n$ every element of $G_{n}$ containing $P$ is a subset of $D$. A space is said to be developable provided it has a development [2]. The developable space $\Sigma$ is said to be complete in sense $C$ or sense A accordingly as it has a decreasingly monotonic development $G_{1}, G_{2}$, $G_{3}, \cdots$ satisfying the first or second of the following conditions: Condition C. If $J$ is an infinite point set and for each $n$ some $g_{n}$ of $G_{n}$ contains all except finitely many points of $J$, then there exists a point $P$ such that every domain containing $P$ contains infinitely many

Presented to the Society, April 19, 1962 under the title Concerning upper semicontinuous collections of mutually exclusive closed and compact point sets; received by the editors August 19, 1963.

1 Principal work on this paper was supported by a National Science Foundation Postdoctoral Fellowship at the University of Texas, 1961-62. Revision and extension were supported in part by the United States Atomic Energy Commission. Reproduction in whole or in part is permitted for any purpose of the U. S. Government. 
points of $J$. Condition A. If $J$ and the $g_{n}$ 's are as above and each $g_{n+1}$ is a subset of $g_{n}$, there exists a point $P$ as above.

The decomposition $G$ of the topological space $\Sigma$ is called upper semicontinuous in Stone's article cited above provided that for each $g$ in $G$ every domain containing $g$ has a subdomain $D$ containing $g$ such that $D$ contains every element of $G$ that it intersects. Stone calls attention to a variation in meaning for this terminology in the literature. The above definition does not require that the decomposition's elements be closed but, if they are, reduces to the definition in [6] for the spaces under consideration here.

Throughout the remainder of this treatment, $\boldsymbol{\Sigma}$ denotes a developable topological space; $G$ denotes an upper semicontinuous decomposition of $\Sigma$, in the above sense, no element of which has a nonbicompact intersection with the closure of its complement; and $I$ denotes the space in which points are the elements of $G$ and regions are the subcollections of $G$ the sums of the elements of which are domains in $\Sigma$. Moore's Axiom 0, which states that every region is a point set, is assumed to hold true in $\Sigma$ with domain, closure, etc., being defined naturally in terms of the notion of region. Following Moore's usage, the notation $K^{*}$ denotes the sum of the sets of the collection $K$. As in [6], no empty set is used. Suitable adjustments in cited definitions may be made where needed on this account.

Theorem 1. I is developable.

Proof. $\Sigma$ has a decreasingly monotonic development. Let $G_{1}, G_{2}$, $G_{3}, \cdots$ denote one satisfying Condition $C$ if $\Sigma$ is complete in sense C and satisfying Condition $A$ if $\Sigma$ is complete in sense A. It may be shown that there exist meanings for the notations $M_{k, h}$ and $U_{k, h}$ for positive integers $k$ and regions $h$ in $I$ such that with respect to a sequence $H_{1}, H_{2}, H_{3}, \cdots$ of well-ordered collections of regions covering $I$ these conditions hold true: (1) For each $k$ and region $h$ of $H_{k}, M_{k, h}$ is a point of $h$ but of no preceding region and $U_{k, h}$ is a collection of elements of $G_{k}$ intersecting $M_{k, h}$. Moreover, if $M_{k, h}$ is not a domain in $\Sigma$ then $U_{k, h}$ is finite and covers both $h^{*}-M_{k, h}$ and $\beta$, the set of all points belonging to $M_{k, h}$ and the boundary of $M_{k, h}$. But if $M_{k, h}$ is a domain it is $h^{*}$. (2) If $k, h$, and $\beta$ are as above, each member $u$ of $U_{k, h}$ contains an element $P$ of $\beta$ such that if $n<k$ and $h^{\prime}$ is the first region of $H_{n}$ containing $M_{k, h}$ then $u$ is a subset of every member of $U_{n, h^{\prime}}$ that contains $P$. (3) If $n<k$ and $g$ belongs to $G$ then the first region of $H_{k}$ containing $g$ is a subset of the first region of $H_{n}$ doing so.

If $A$ belongs to the region $R$ in $I$ and is a domain in $\Sigma$ there exist an teger $s$ and element $P$ of $A$ such that every member of $G_{s}$ containing 
$P$ is a subset of $A$. If $i \geqq s$ and the region $h$ of $H_{i}$ contains $A$ then $A$ is $M_{i, h}$, for if it is not then some element of $U_{i, h}$ contains $P$ and is therefore a subset of $A$ intersecting $M_{i, h}$. Hence $h^{*}$ is $A$ and $h$ is a subset of $R$. If $A$ is not a domain, then for each $n$ there exists a first region $h_{n}$ of $H_{n}$ containing $A$. Let $T_{n}$ denote $U_{n, h_{n}}$ and let $C_{n}$ denote $M_{n, h_{n}}$. By condition (1) above, the collections $T_{n}$ are finite and cover $\beta$, the intersection of $A$ and the closure of the complement of $A$. Thus for some $n$ there exists a subcollection $V$ of $T_{1}+\cdots+T_{n}$ covering the bicompact point set $\beta$ such that $V^{*}$ is a subset of $R^{*}$. Let $Y$ denote a point of $\beta$ and let $R^{\prime}$ denote a region in $I$ containing $A$ such that $R^{\prime *}$ is a subset of $V^{*}+A$. There exists some $k>n$ such that every element of $G_{k}$ containing $Y$ is a subset of $R^{\prime *}$. Some element of $T_{k}$ contains $Y$ and by condition (1) all of the elements of $T_{k}$ intersect $C_{k}$. Hence $C_{k}$ intersects $R^{\prime *}$ and is therefore a subset of $V^{*}+A$. With the use of condition (3) it may be seen that $h_{1}, h_{2}, h_{3}, \cdots$ is decreasingly monotonic, hence that each $C_{i+1}$ lies in $h_{i}$. But no predecessor of $h_{i}$ contains $A$ and thus condition (3) shows that $h_{i}$ is the first region of $H_{i}$ containing $C_{i+1}$. By condition (2), each member $t$ of $T_{\boldsymbol{k}}$ contains an element $P$ of $C_{k}$ belonging to the boundary of $C_{k}$ in $\Sigma$ such that if $i \leqq n$, then $t$ is a subset of every member of $T_{i}$ that contains $P$. Clearly, this implies that $T_{\mathbf{k}}^{*}$ is a subset of $V^{*}$. By condition (1), $h_{\mathbf{k}}^{*}$ is a subset of $T_{\mathbf{k}}^{*}+C_{k}$. From these facts it follows that $h_{k}$ is a subset of $R$. For some $s>k$ the members of $G_{s}$ containing $Y$ are subsets of $h_{k}^{*}$. If $i \geqq s$ and the region $h$ of $H_{i}$ contains $A$ then some element $u$ of $U_{i, h}$ contains $Y$ and is therefore a subset of $h_{\mathbf{k}}^{*}$. Since $M_{i, h}$ intersects $u$ it belongs to $h_{k}$. So $h_{k}$ does not precede the first region $h^{\prime}$ of $H_{k}$ containing $M_{i, h}$. By condition (3) the region $h$ is a subset of $h^{\prime}$, which therefore contains $A$ and does not precede $h_{k}$. Thus $h^{\prime}$ is $h_{k}$ and $h$ is a subset of $R$. It follows that $H_{1}, H_{2}, H_{3}, \cdots$ is a development of $I$.

TheOREM 2. If $\Sigma$ is complete in sense $\mathrm{C}$, so is $I$.

Proof. With $G_{1}, G_{2}, G_{3}, \cdots$ and $H_{1}, H_{2}, H_{3}, \cdots$ as above it follows that if for each $n, W_{n}$ denotes $H_{n}+H_{n+1}+H_{n+2}+\cdots$ then $W_{1}, W_{2}$, $W_{3}, \cdots$ is a decreasingly monotonic development of $I$. If $G^{\prime}$ is an infinite subcollection of $G$ and for each $n$, some element $w_{n}$ of $W_{n}$ contains all except finitely many elements of $G^{\prime}$, then for each $n$ there exists a first region $h_{n}$ of $H_{n}$ such that some $w_{i}$ is a subset of $h_{n}$. Let $J$ denote an infinite subset of $G^{\prime *}$ such that no member of $G^{\prime}$ contains two elements of $J$. In view of condition (1) of the above proof it is clear that for each $n$ the collection $U_{n, h_{n}}$ is finite, and hence there is a collection $T_{n}$ of all of its elements having an infinite intersection with $J$. Moreover, it follows inductively that for each $n$ there is a collection 
$F_{n}$ of all $n$-term sequences $f$ such that (1) if $i \leqq n$, the $i$ th term of $f$ belongs to $T_{i},(2)$ some infinite subset of $J$ intersects each term of $f$, and (3) if for each $i>1$ every element of $T_{i}$ is a subset of some element of $T_{i-1}$ then $f$ is decreasingly monotonic. For each $n>1$ and sequence $f$ in $F_{n}$ there exists a sequence $f^{\prime}$ in $F_{n-1}$ such that if $i \leqq n-1$, the $i$ th term of $f^{\prime}$ is the $i$ th term of $f$. Since each collection $F_{n}$ is finite, there exists a sequence $f_{1}, f_{2}, f_{3}, \cdots$ such that, for each $n, f_{n}$ belongs to $F_{n}$, and if $n>1$ and $i \leqq n-1$, the $i$ th term of $f_{n-1}$ is the $i$ th term of $f_{n}$. There exists a nonrepeating sequence $P_{1}, P_{2}, P_{3}, \cdots$ such that for each $n, P_{n}$ belongs to $J$ and every term of $f_{n}$. It follows inductively that if for each $n, R_{n}$ denotes the $n$th term of $f_{n}$, then each $R_{n}$ contains all except finitely many terms of $P_{1}, P_{2}, P_{3}, \cdots$. Each $R_{n}$ belongs to $G_{n}$ and thus in view of the conditions on $G_{1}, G_{2}, G_{3}, \cdots$ it may be seen that some element $M$ of $G$ contains a point $X$ such that every domain in $\Sigma$ to which $X$ belongs contains infinitely many of the points $P_{n}$. If the domain $R$ in $I$ contains $M$ then it has an infinite intersection with $G^{\prime}$, for no set in this collection contains two of the points $P_{n}$ and $R^{*}$ is a domain in $\Sigma$.

\section{TheOREM 3. If $\Sigma$ is complete in sense A, so is $I$.}

Proof. Retaining the above notation and requiring that $w_{1}, w_{2}$, $w_{3}, \cdots$ be decreasingly monotonic we see that if $n>1$ there exists some $i>n$ such that $w_{i}$ is a subset of $h_{n}$ and $h_{n-1}$. For some $k \geqq i, w_{i}$ is an element $h$ of $H_{k}$. By condition (3) in the proof of Theorem $1, h$ is a subset of the first region $h^{\prime}$ of $H_{n}$ containing $M_{k, h}$. No term of $w_{1}$, $w_{2}, w_{3}, \cdots$ is a subset of a predecessor of $h_{n}$ and the point $M_{k, h}$ lies in $h_{n}$. So $h^{\prime}$ is $h_{n}$. Similarly, $h_{n-1}$ is the first element of $H_{n-1}$ containing $M_{k, h}$. Hence, by condition (3), $h_{n}$ is a subset of $h_{n-1}$ and this, for analogous reasons, implies that $h_{n-1}$ is the first element of $H_{n-1}$ containing $M_{n, h_{n}}$. Therefore, by condition (2), each element of $T_{n}$ is a subset of some element of $T_{n-1}$. So by definition of the collections $F_{n}$ each $R_{n+1}$ is a subset of $R_{n}$. Thus $G$ contains an element $M$ as in the above proof.

Consequences. Terminology appearing below that is not explicitly cited or defined here is much as in [4].

A space satisfies Axiom $1_{3}$ of [6] if and only if it is a regular $T_{1}$ space $\Sigma$ as in the hypothesis of Theorem 1. A space satisfies Axiom 1 if and only if it is a regular $T_{1}$ space $\Sigma$ as in the hypothesis of Theorem 2 . In these spaces, a closed point set is compact [1] if and only if it is bicompact [6, Chapter 1]. So if $\Sigma$ satisfies Axiom $1_{3}, I$ is regular and $T_{1}$. Moreover, it may be seen that if $\beta$ is the boundary of an element 
of an upper semicontinuous decomposition of a regular $T_{1}$ space and the decomposition space satisfies the first axiom of countability, then $\beta$ is bicompact if and only if every collection of domains covering $\beta$ is refined by a point countable collection of domains covering $\beta$. Thus the following theorem is a corollary to Theorems 1 and 2.

CoROllary 1. If (1) $U$ is an upper semicontinuous decomposition of a space satisfying Axioms 0 and $1_{3}$ (Axioms 0 and 1) and (2) any collection of domains covering the boundary $\beta$ of an element of $U$ not a domain is refined by a point countable collection of domains covering $\beta$, these conditions are equivalent:

(1) The decomposition space satisfies Axiom $1_{3}$ (Axiom 1).

(2) The decomposition space satisfies the first axiom of countability.

(3) No element of $U$ has a noncompact boundary.

The hypothesis of Corollary 1 involving point countable refinements is nonsuperfluous: There exists an upper semicontinuous decomposition of a certain space satisfying Axioms 0 and 1 such that, while the decomposition space is metrizable and compact, the decomposition contains an element with a noncompact boundary [6, (implicitly) p. 66].

Normalcy is preserved by every upper semicontinuous decomposition of a normal space. Moreover, if $\beta$ is the boundary of an element of an upper semicontinuous decomposition of a normal $T_{1}$ space $S$ and the decomposition space satisfies the first axiom of countability, then $\beta$ is compact [7]. If, additionally, $S$ satisfies Axiom $1_{3}, \beta$ is bicompact. So the following theorem is a corollary to Theorems 1 and 2 .

CoROllaRY 2. If $U$ is an upper semicontinuous decomposition of a normal space satisfying Axioms 0 and $1_{3}$ (Axioms 0 and 1 ), these conditions are equivalent:

(1) The decomposition space is a normal space satisfying Axioms $1_{3}$ (Axiom 1).

(2) The decomposition space satisfies the first axiom of countability.

(3) No element of $U$ has a noncompact boundary.

Collectionwise normalcy [2] is preserved by upper semicontinuous decompositions of collectionwise normal spaces. Every collectionwise normal space satisfying Axioms 0 and $1_{3}$ is metrizable [2]. Every metrizable space complete in sense $C$ is metrically topologically complete [9]. The next theorem follows from these theorems and Corollary 2.

Corollary 3 (Morita-Hanai [7, Theorem 1] and A. H. Stone [12, Theorem 1]; I. A. VaĬnŠtěIN [13, Theorem 6]). If $U$ is an 
upper semicontinuous decomposition of a space that is metrizable (metrically topologically complete), these conditions are equivalent:

(1) The decomposition space is metrizable (metrically topologically complete).

(2) The decomposition space satisfies the first axiom of countability.

(3) No element of $U$ has a noncompact boundary.

The theorem of Morita-Hanai-Stone may also be derived from Corollary 1, the paracompactness of the closed continuous images of paracompact Hausdorff spaces [5], and the Nagata-Smirnov theorem $[8],[11]$.

\section{REFERENCES}

1. P. S. Alexandroff and P. Urysohn, Memoire sur les espaces topologiques compacts, Verh. Nederl. Akad. Wetensch. Afd. Naturk. Sect. I, 14 (1929), no. 1, 1-96. 186.

2. R. H. Bing, Metrization of topological spaces, Canad. J. Math. 3 (1951), 175-

3. M. Fréchet, Sur quelques points du calcul fonctionnel, Rend. Circ. Mat. Palermo 22 (1906), 1-74.

4. J. L. Kelley, General topology, Van Nostrand, New York, 1955.

5. E. Michael, Another note on paracompact spaces, Proc. Amer. Math. Soc. 8 (1957), 822-828.

6. R. L. Moore, Foundations of point set theory, rev. ed., Amer. Math. Soc. Colloq. Publ. Vol 13, Amer. Math. Soc., Providence, R. I., 1962.

7. K. Morita and S. Hanai, Closed mappings and metric spaces, Proc. Japan Acad. 32 (1956), 10-14.

8. J. Nagata, On a necessary and sufficient condition of metrizability, J. Inst. Polytech. Osaka City Univ. Ser. A. 1 (1950), 93-100.

9. J. H. Roberts, A property related to completeness, Bull. Amer. Math. Soc. 38 (1932), 835-838.

10. Mary Ellen Estill Rudin, Concerning abstract spaces, Duke Math. J. 17 (1950), 317-327.

11. Yu. M. Smirnov, A necessary and sufficient condition for metrizability of a topo. logical space, Dokl. Akad. Nauk SSSR 77 (1951), 197-200.

12. A. H. Stone, Metrizability of decomposition spaces, Proc. Amer. Math. Soc. 7 (1956), 690-700.

13. I. A. VałnšteY̌n, On closed mappings of metric spaces, Dokl. Akad. Nauk SSSR 57 (1947), 319-321. (Russian)

The University of Texas and

Sandu Corporation 